\title{
The Study on the sEMG Signal Characteristics of Muscular Fatigue Based on the Hilbert-Huang Transform ${ }^{\star}$
}

\author{
Bo Peng ${ }^{1,2}$, Xiaogang $\mathrm{Jin}^{2, \star \star}$, Yong $\mathrm{Min}^{2}$, and Xianchuang $\mathrm{Su}^{3}$ \\ ${ }^{1}$ Ningbo Institute of Technology, Zhejiang university, \\ Ningbo 315100, China \\ 2 AI Institute, College of Computer Science, \\ Zhejiang university, Hangzhou 310027, China \\ xiaogangj@cise.zju.edu.cn \\ ${ }^{3}$ College of Software Engineering, Zhejiang university, \\ Hangzhou 310027, China
}

\begin{abstract}
Muscular fatigue refers to temporary decline of maximal power ability or contractive ability for muscle movement system. The signal of surface electromyographic signal (sEMG) can reflect the changes of muscular fatigue at certain extent. In many years, the application of signal of sEMG on evaluation muscular fatigue mainly focus on two aspects of time and frequency respectively. The new method HilbertHuang Transform(HHT) has the powerful ability of analyzing nonlinear and non-stationary data in both time and frequency aspect together. The method has self-adaptive basis and is better for feature extraction as we can obtain the local and instantaneous frequency of the signals. In this paper, we chose an experiment of the static biceps data of twelve adult subjects under the maximal voluntary contraction (MVC) of $80 \%$. The experimental results proved that this method as a new thinking has an obvious potential for the biomedical signal analysis.
\end{abstract}

\section{Introduction}

Muscular fatigue [1] is an exercise-induced reduction in maximal voluntary muscle force. It may arise not only because of peripheral changes at the level of the muscle, but also because the central nervous system fails to drive the motoneurons adequately. The measurement of the muscular fatigue is according to the decline of the system maximal function. The adaptation of the neuromuscular system to heavy resistance exercise is a very complex result of many factors. This implies central and peripheral neural adaptations as well as chemical and morphological modifications of muscle tissue.

Surface electromyographic signal (sEMG) is an one-dimensional time series signal of neuromuscular system that recorded for skin surface, the time-frequency

\footnotetext{
* Supported by Zhejiang Provincial Natural Science Foundation of China under Grant No. Y105697 and Ningbo Natural Science Foundation (2005A610004).

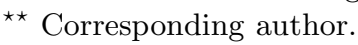


domain and nonlinear dynamical characters of it are sensitive to the intensity and state for muscular activities and therefore it is a valuable method for muscle functional evaluation. For many years, the analysis of sEMG concentrated on two main fields, time domain and the frequency domain [2]. The common use way of traditional time domain analysis is to regard the sEMG signal as the function of time, using some indicators like Integrated EMG (IEMG) or some statistical features such as Root Mean Square(RMS). In aspect of spectrum analysis, the typical method is the Fast Fourier Transform short as FFT. The spectrum we acquire from the FFT can show the distribution of the signals in different frequency component quantitatively. In order to portray the characteristic of the spectrum, researchers often use the following two indicators: Median Frequency (MF) and Mean Power Frequency (MPF) [3].

Most research works have found that the indicators of frequency analysis of the muscles in limbs and waist have good regularity in the condition of the static burthen. The main manifestation is the decline of the MPF or MF and the increase of power spectrum or the ratio of the low/high frequency during the process of fatiguing [4] [5]. However, under the dynamic condition, the alteration of the MPF and MF during the fatiguing process exist considerable difference from which can hardly get a universal conclusion. On account of above, we chose the static biceps data which have remarkable features of fatiguing to test our new method's performance in this paper.

\section{The Method of Hilbert - Huang Transform}

A new method [6] for analyzing nonlinear and non-stationary data has been developed in 1998 by N. E. Huang and others who lately made some amelioration on this method [7] in 1999. The key part of the method is the Empirical Mode Decomposition (EMD) method with which any complicated data set can be decomposed into a finite and often small number of Intrinsic Mode Functions (IMF) that admit well-behaved Hilbert transforms. This decomposition method is adaptive, and, therefore, highly efficient. Since the decomposition is based on the local characteristic time scale of the data, it is applicable to nonlinear and non-stationary processes. With the Hilbert transform, the IMF functions yield instantaneous frequencies as functions of time that give sharp identifications of imbedded structures. The final presentation of the results is the energy - frequency - time distribution, designated as the Hilbert spectrum. In this method, the main conceptual innovations are the introduction of IMF based on local properties of the signal, which makes the instantaneous frequency meaningful.

The method of Hilbert - Huang Transform (HHT) is composed of two parts: first one is the process of EMD which generate the products called IMF, and the second one it the traditional Hilbert transform and analysis on these IMF. IMF is a function that satisfies two conditions: (1) in the whole data set, the number of extremal and the number of zero crossings must either equal or differ at most by one; and (2) at any point, the mean value of the envelope defined by the local maxima and the envelope defined by the local minima is zero. 
Find out all the local maxima and minima of the original signal. Once the extremal are identified, all the local maxima are connected by a cubic spline line as the upper envelope. Repeat the procedure for the local minima to produce the lower envelope. The upper and lower envelopes should cover all the data between them. Their mean is designated as $\mathrm{m} 1$, and the difference between the data and $m_{1}$ is the first component, $h_{1}$.

$$
X(t)-m_{1}=h_{1}
$$

Then, we have to judge whether $h_{1}$ be the IMF according to the requirements mentioned above. If not satisfy, the sifting process has to be repeated more times. In the second sifting process, $h_{1}$ is treated as the data, then,

$$
h_{1}-m_{11}=h_{11}
$$

We can repeat this sifting procedure $k$ times, until $h_{1 k}$ is an IMF, that is:

$$
h_{1(k-1)}-m_{1 k}=h_{1 k}
$$

Then, it is designated as:

$$
c_{1}=h_{1 k}
$$

The $c_{1}$ is the IMF component for the original signal. We can separate $c_{1}$ from the rest of the data by

$$
X(t)-c_{1}=r_{1}
$$

Since the residue, $r_{1}$, still contains information of longer period components, it is treated as the new data and subjected to the same sifting process as described above. This procedure can be repeated on all the subsequent $r_{j} \mathrm{~s}$, and the result is:

$$
r_{1}-c_{2}=r_{2}, \cdots, r_{n-1}-c_{n}=r_{n}
$$

The sifting process can be stopped, when the residue, $r_{n}$, becomes a monotonic function from which no more IMF can be extracted. By summing up equations (5) and (6), we finally obtain

$$
X(t)=\sum_{i=1}^{n} c_{i}+r_{n}
$$

Having obtained the intrinsic mode function components, we will have no difficulties in applying the Hilbert transform to each component, and computing the instantaneous frequency according to the following steps. For an arbitrary time series, $X(t)$, we can always have its Hilbert Transform, $Y(t)$, as

$$
Y(t)=\frac{1}{\pi} P \int_{-\infty}^{+\infty} \frac{X\left(t^{\prime}\right)}{t-t \prime} \mathrm{d} t^{\prime}
$$

where $P$ indicates the Cauchy principal value. With this definition, $X(t)$ and $Y(t)$ form the complex conjugate pair, so we can have an analytic signal, $Z(t)$, as

$$
Z(t)=X(t)+\mathrm{i} Y(t)=a(t) \mathrm{e}^{\mathrm{i} \theta(t)}
$$


in which we can also write as

$$
\begin{gathered}
a(t)=\left[X^{2}(t)+Y^{2}(t)\right]^{1 / 2} \\
\theta(t)=\arctan \left(\frac{Y(t)}{X(t)}\right)
\end{gathered}
$$

and the instantaneous frequency defined as

$$
\omega=\frac{\mathrm{d} \theta(t)}{\mathrm{d} t}
$$

After performing the Hilbert transform on each IMF component, we can express the data in the following form:

$$
X(t)=\sum_{j=1}^{n} a_{j}(t) \exp \left(\mathrm{i} \int \omega_{j}(t) \mathrm{d} t\right)
$$

The equation also enables us to represent the amplitude and the instantaneous frequency as functions of time in a three-dimensional plot, in which the amplitude can be contoured on the frequency - time plane. This frequency- time distribution of the amplitude is designated as the Hilbert amplitude spectrum, $H(\omega ; t)$, or simply Hilbert spectrum. If amplitude squared is more desirable commonly to represent energy density, then the squared values of amplitude can be substituted to produce the Hilbert energy spectrum just as well.

With the Hilbert spectrum defined, we can also define the marginal spectrum, $h(\omega)$, as

$$
h(\omega)=\int_{0}^{T} H(\omega, t) \mathrm{d} t
$$

In addition to the marginal spectrum, we can also define the instantaneous energy density level, IE, as

$$
I E(t)=\int_{\omega} H^{2}(\omega, t) \mathrm{d} \omega
$$

and the marginal energy spectrum ES, as

$$
E S(\omega)=\int_{0}^{T} H^{2}(\omega, t) \mathrm{d} t
$$

Although the HHT is a powerful method for analyzing nonlinear and nonstationary data, it has deficiencies also. Some research work has been done to ameliorate this [8. However, the application of HHT in many fields have proved its performance exceed the traditional one-dimensional time series signals analysis methods [9] 10] 11].

\section{$3 \quad$ Method and Result}

\section{$3.1 \quad$ Subjects}

Twelve healthy adults of male university students, age ranging between 19 and 24, participates in this study. Subjects were all right hand dominant. They had no fierce movement before the test. 


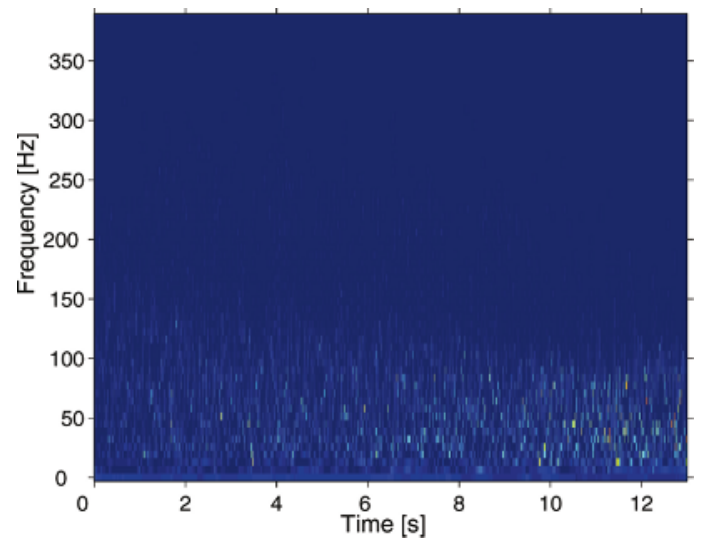

Fig. 1. The Hilbert spectrum of a sample sEMG ( $80 \% \mathrm{MVC})$ after the EMD process

\subsection{Procedures}

Subjects were first allowed to warm up the biceps. Then the wrists of the subjects were burdened with a weight of $80 \%$ MVC until they exhausted. During the procedures, signals were recorded with the sampling frequency of $1000 \mathrm{~Hz}$.

\subsection{Results and Analysis}

We deal with the data by the HHT, and Fig.1 shows the energy - frequency time distribution of a sample sEMG ( $80 \%$ MVC) which we continue to use in Fig.2 and Fig.3, designated as the Hilbert spectrum. The brighter denote the higher energy and the darker denote the lower. Roughly, we can see from the Fig. 1 the frequency distribution is wide at the beginning and concentrated to the lower frequency at the end, and in Fig.1, We can also see the energy became higher by the time variable for the right part of the spectrum is brighter than the

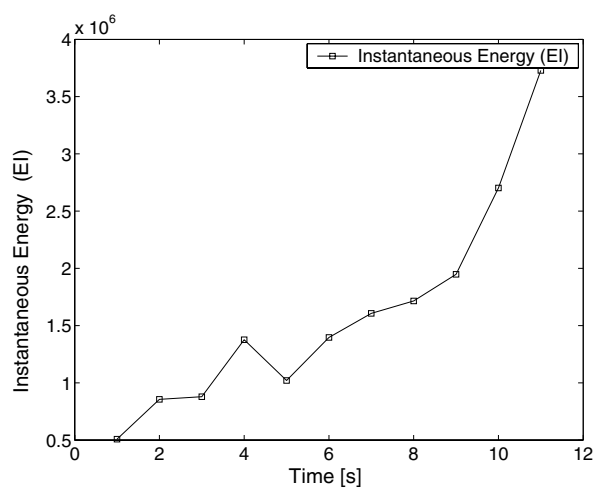

(a)

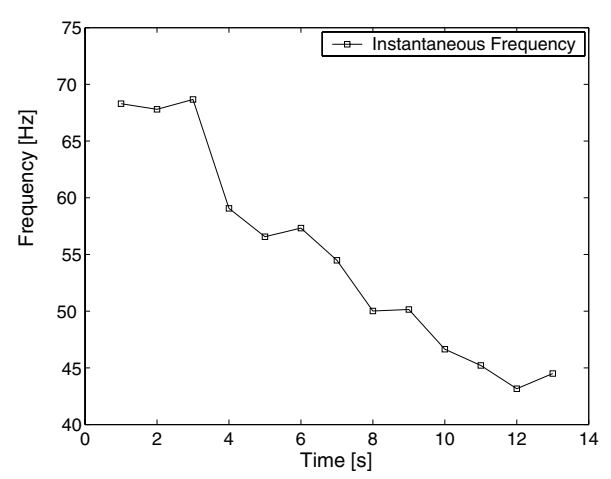

(b)

Fig. 2. (a)The instantaneous energy, IE the sample of the sample (b)The instantaneous frequency of the sample 
left. We draw a conclusion qualitatively that during the process of the muscle fatigue test, the frequency is going down and the energy is increasing. We get more detailed and obvious features from further analysis below.

Fig.2(a) shows the changing trend of instantaneous energy (IE) by the factor of time. We can see the energy approximately go up during the whole fatiguing process. In Fig.2(b), we can see clearly that the instantaneous frequency is going down during the fatiguing process. To calculate the instantaneous frequency, please consult formulas. The ordinary way is to use the indicator MF or MPF [3]. During the fatiguing course, the MF is move leftward, means the frequency deceases generally. So, our results is consistent with the traditional approachs and more convenient and evident 13 .

In order to contrast, we extract the first second and last second of the signals to test together, which we can see even more obviously the changes by fatiguing. In the following analyzing, we use the average $80 \% \mathrm{MVC}$ data of the twelve whole subjects.

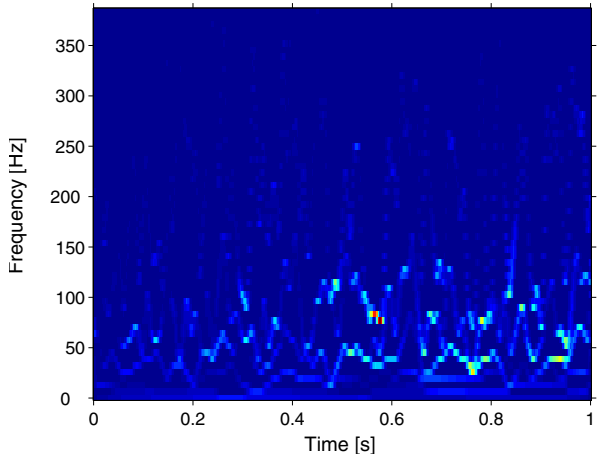

(a)

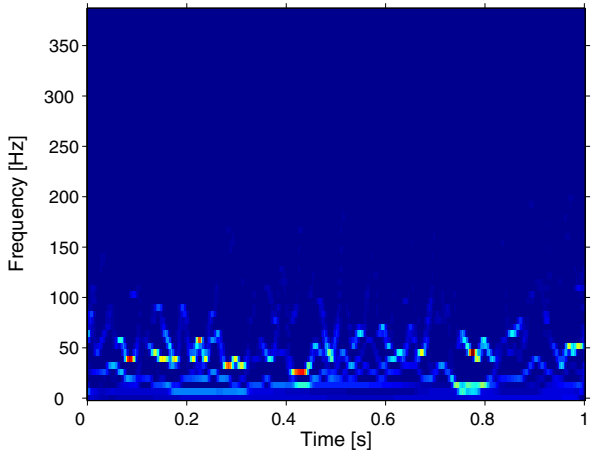

(b)

Fig. 3. The first second (a) and the last second (b)of the Hilbert spectrum of the average twelve subjects' sEMG $(80 \% \mathrm{MVC})$ after the EMD process

Fig.3(a) and Fig.3(b) show the Hilbert spectrum of the first second and the last second of the average samples. Because the experiment was ended when the subjects were exhausted, so we can consider the muscles were in the state of fatigue in the last second. In contrast to Fig.3(a), frequency is concentrate to the lower and the energy is higher in Fig.3(b). Although Fig.3(a) and Fig.3(b) is the small sections of the Fig.1 in which they amplified the features of fatigue.

Fig.4(a) and Fig.4(b) show the energy's distribution on the frequency. We can see the frequency of the last second is concentrate to the lower frequency relatively and the energy is much higher than the first second's. This alteration is even much remarkable in Fig.4(c) and Fig.4(d) which show the varying by the time variable. 


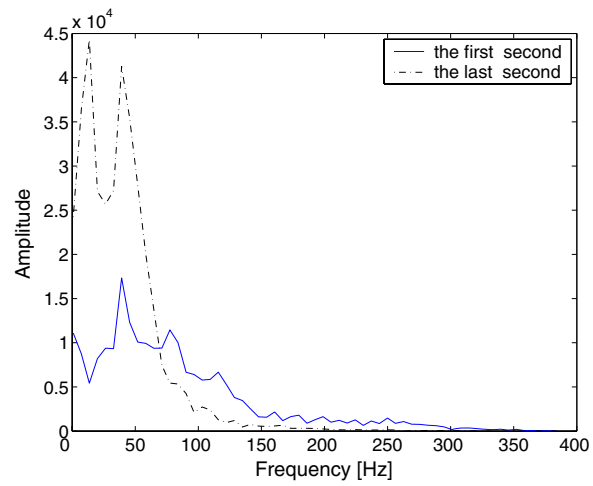

(a)

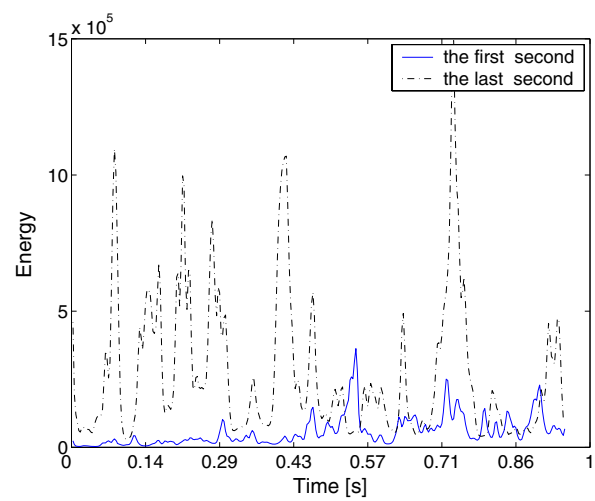

(c)

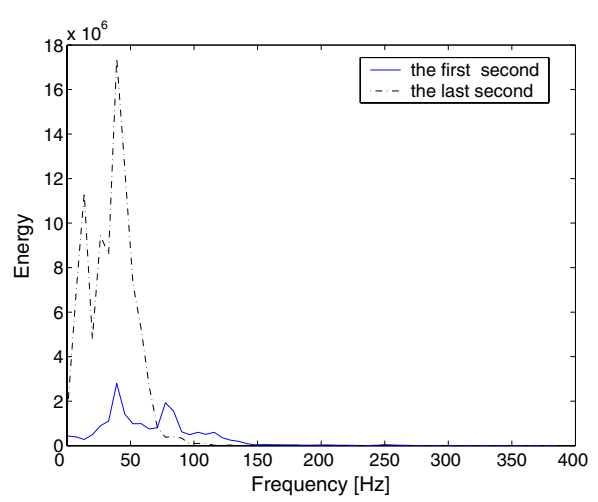

(b)

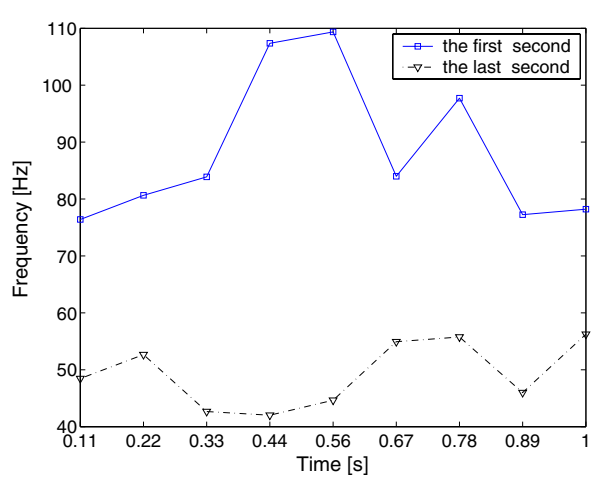

(d)

Fig. 4. (a)The Hilbert marginal spectrum,(b)the Marginal energy spectrum ES, (c)the instantaneous energy density level, IE, and(d) the instantaneous frequency of the first and the last second of average twelve subjects' sEMG (80\%MVC)

\section{Conclusion}

In this paper, we adopted a new method-HHT, which is good at processing nonlinear and non-stationary data in two dimensions of both time domain and frequency domain to research the sEMG signal to find the features of muscular fatigue. We choose twelve adult university students as the experimental subjects. All the results we gained above are very consistent with the experiential conclusion that the frequency is going down and the energy is going up in the process of muscular fatiguing in [4 5. The results also have proved that the HHT method has good performance and massive potential in analyzing the biomedical signals. However, the results in this paper are preliminary exploration, more works have to be done in the furture, even the HHT itself has some defects to ameliorate. Anyway, the HHT method has showed the extensive applicable foreground. 


\section{References}

1. Gandevi S. C.: Spinal and supraspinal factors in human muscle fatigue. J. Physiological reviews. 81 (2001) $1725-1789$

2. Hagg G. M.: Interpretation of EMG spectral alternations and alternation indexes at sustained contraction. J. Appl. Physiol. 73 (1992) 1211-1217

3. Bilodeau M., Schindler-Ivens S., Williams D. M., Chandran R., Sharma S S.: EMG frequency content changes with increasing force and during fatigue in the quadriceps femoris muscle of men and women. J. Electromyography and Kinesiology. 13 (2003) 83-92

4. Mannion A. F., Connelly B., Wood K.: Electromyographic median frequency changes during isometric contraction of the back extensors to fatigue. Spine. 19 (1994) 1223-1229

5. Kazumi M., Tadashi M., Tsugutake S.: Changes in EMG parameters during static and dynamic fatiguing contractions. J. Electromyography and Kinesiology. 9 (1999) 9-46

6. Huang N. E., Shen Z., Long S. R.: The empirical mode decomposition and Hilbert spectrum for nonlinear and non-stationary time series analysis. J. Proc. R. Soc. London. 454 (1998) 899-995

7. Huang N. E., Shen Z., Long S. R.: A new view of non linear water waves: the Hilbert spectrum. J. Ann. Rev. Fluid Mech. 31 (1999) 417-457

8. Peng Z. K., Peter W .T., Chu F. L.: A comparison study of improved HilbertHuang transform and wavelet transform: Application to fault diagnosis for rolling bear. Mechanical Systems and Signal Processing. 19 (2005) 974-988

9. Dean G. D.: The Application of Hilbert-Huang Transforms to Meteorological Datasets. J. of Atmospheric and Oceanic Technology. Boston. 21 (2004) 599-612

10. Zhang R. R., VanDemark L., Liang L., Hu Y.: On estimating site damping with soil non-linearity from earthquake recordings. International Journal of Non-Linear, Mechanics. 39 (2004) 1501-1517

11. Phillips S. C., Gledhill R. J., Essex J. W., Edge C. M.: Application of the HilbertHuang Transform to the analysis of molecular dynamic simulations. J. Phys. Chem. A. 107 (2003) 4869-4876

12. Loh C. H., Wu T. C., Huang N. E.: Application of EMD+HHT method to identify near-fault ground motion characteristics and structural responses. BSSA. Special Issue of Chi-Chi Earthquake. 91 (2001) 1339-1357

13. Yang B. Z., Suh C. S.: Interpretation of crack-induced rotor non-linear response using instantaneous frequency. Mechanical Systems and Signal Processing. 18 (2004) 491-513 